\title{
BMJ Open Qualitative perspectives on the sustainability of sexual health continuous quality improvement in clinics serving remote Aboriginal communities in Australia
}

\author{
Praveena Gunaratnam, ${ }^{1}$ Gill Schierhout, ${ }^{1,2}$ Jenny Brands, ${ }^{3}$ Lisa Maher, ${ }^{1,4}$ \\ Ross Bailie, ${ }^{5}$ James Ward, ${ }^{6}$ Rebecca Guy, ${ }_{1}^{1}$ Alice Rumbold, ${ }^{7}$ Nathan Ryder, ${ }^{1,8,9}$ \\ Christopher K Fairley, ${ }^{10,11}$ Basil Donovan, ${ }^{1,12}$ Liz Moore, ${ }^{13}$ John Kaldor, ${ }^{1}$ \\ Stephen Bell ${ }^{1,14}$
}

To cite: Gunaratnam $P$, Schierhout G, Brands J, et al. Qualitative perspectives on the sustainability of sexual health continuous quality improvement in clinics serving remote Aboriginal communities in Australia. BMJ Open 2019;9:e026679. doi:10.1136/ bmjopen-2018-026679

Received 14 September 2018 Revised 20 February 2019 Accepted 4 April 2019
Check for updates

(c) Author(s) (or their employer(s)) 2019. Re-use permitted under CC BY-NC. No commercial re-use. See rights and permissions. Published by BMJ.

For numbered affiliations see end of article.

Correspondence to Dr. Stephen Bell; sbell@kirby.unsw.edu.au

\section{ABSTRACT}

Objectives To examine barriers and facilitators to sustaining a sexual health continuous quality improvement (CQI) programme in clinics serving remote Aboriginal communities in Australia.

Design Qualitative study.

Setting Primary health care services serving remote Aboriginal communities in the Northern Territory, Australia.

Participants Seven of the 11 regional sexual health coordinators responsible for supporting the Northern Territory Government Remote Sexual Health Program. Methods Semi-structured in-depth interviews conducted in person or by telephone; data were analysed using an inductive and deductive thematic approach.

Results Despite uniform availability of CQI tools and activities, sexual health CQI implementation varied across the Northern Territory. Participant narratives identified five factors enhancing the uptake and sustainability of sexual health CQI. At clinic level, these included adaptation of existing CQI tools for use in specific clinic contexts and risk environments (eg, a syphilis outbreak), local ownership of CQI processes and management support for CQI. At a regional level, factors included the positive framing of CQI as a tool to identify and act on areas for improvement, and regional facilitation of clinic level CQI activities. Three barriers were identified, including the significant workload associated with acute and chronic care in Aboriginal primary care services, high staff turnover and lack of Aboriginal staff. Considerations affecting the future sustainability of sexual health CQI included the need to reduce the burden on clinics from multiple CQI programmes, the contribution of regional sexual health coordinators and support structures, and access to and use of high-quality information systems.

Conclusions This study contributes to the growing evidence on how CQI approaches may improve sexual health in remote Australian Aboriginal communities. Enhancing sustainability of sexual health CQI in this context will require ongoing regional facilitation, efforts to build local ownership of CQI processes and management of competing demands on health service staff.

\section{Strengths and limitations of this study}

- This is the first qualitative study to document clinic-level and regional-level factors influencing uptake and sustainability of a sexual health continuous quality improvement (CQI) programme in government-run primary care services serving remote Aboriginal communities in the Northern Territory (NT), Australia.

- Using in-depth interviews with 7 of the 11 sexual health coordinators employed to improve testing and treatment for sexually transmissible infections (STIs) in government-run primary cares services elicited rich data about the challenges influencing sustained use of a sexual health CQI approach to improve STI testing and treatment rates.

- This is a qualitative study with a small, non-random sample size and as such findings should not be viewed as generalisable to other settings.

\section{INTRODUCTION}

Residents in remote Aboriginal and Torres Strait Islander (hereafter referred to as 'Aboriginal') communities in Australia experience high rates of sexually transmissible infections (STIs), estimated in one large, multi-community study at $10 \%, 11 \%$ and $18 \%$, respectively, for gonorrhoea, chlamydia and syphilis. ${ }^{1}$ Clinical guidelines for these communities recommend at least annual STI testing, timely treatment, partner notification and regular re-testing, particularly those under 35 years old. ${ }^{2}{ }^{3}$ However, recent estimates indicate that annual STI testing coverage in remote communities is still only $20 \%$, with lower rates in men than in women. ${ }^{4}$

Since the 1990s, continuous quality improvement (CQI) approaches-typically 
consisting of 'plan-do-study-act' cycles to monitor the functioning of organisational systems and identify and implement improvements ${ }^{5}$ - have been used to improve health care quality and outcomes associated with, for example, chronic disease. ${ }^{6-10}$ In Australia, Aboriginal Community Controlled Health Services (ACCHS) and government-run health services providing primary health care to Aboriginal communities have used CQI extensively to make evidence-based improvements in clinical care. ${ }^{11-13}$

Between 2011 and 2014, the STIs in Remote communities, ImproVed and Enhanced primary care (STRIVE) trial was conducted in 67 remote communities in the Northern Territory (NT), Queensland and Western Australia. ${ }^{14}$ STRIVE demonstrated that a clinic level sexual health CQI programme supported by regional dedicated sexual health coordinators ${ }^{14-16}$ could improve STI testing rates, ${ }^{15}$ with aspects of the CQI programme becoming normalised in clinical practice. ${ }^{16}$ However, testing increases were from a low baseline and did not lead to lower STI prevalence over the time frame of the trial. $^{15}$

Elements of the STRIVE CQI approach were incorporated into the NT Government Remote Sexual Health Program. ${ }^{3}$ The programme employs regionally-based sexual health coordinators to work with government-run primary care services to improve STI testing and treatment. Their roles include providing feedback on STI control through audits and review of clinical data; annual discussions with staff to assess and make recommendations for improving clinic-wide systems that support STI control (systems assessments); and sexual health education and staff training. ${ }^{3}$

To gain insight into CQI practice for STI control in the NT since the STRIVE study finished in 2014, and to explore factors affecting the long-term sustainability of this approach in government-run health services, we conducted qualitative interviews with sexual health coordinators. Data were collected as part of a new study called STRIVEplus-a long-term observational study of sexual health CQI in the NT between 2015 and 2019.

\section{METHODS}

This was a qualitative study using semi-structured, in-depth interviews. The design, data collection, analysis and reporting of this study were conducted in accordance with the Standards for Reporting Qualitative Research. ${ }^{17}$

\section{Setting}

The NT of Australia covers 1.4 million square kilometres, with an estimated resident population of 230000 , of whom $26 \%$ are Aboriginal (compared with $3 \%$ nationally). ${ }^{18}$ Most (79\%) Aboriginal residents in the NT live in remote areas ${ }^{19}$ and are served by two types of primary care services-ACCHS or NT government health services. Remote clinics are primarily staffed by Aboriginal health workers or registered nurses (often on short-term fly in-fly out contracts) with support from resident or visiting general practitioners and other specialist or allied health providers. ${ }^{16}$

The setting for this study was the NT government health service sector, specifically the NT Government Remote Sexual Health Program. This programme coordinates support for sexual health service delivery across both the Top End Health Service (covering approximately 35\% of the geographic area and $80 \%$ of the population) and Central Australia Health Service (65\% of the geographic area and $20 \%$ of the population). ${ }^{19}$

\section{Study participants}

All sexual health coordinators working in the NT Department of Health $(n=11)$ in 2016 were approached up to three times and invited to participate in an in-depth qualitative interview. This resulted in seven sexual health coordinators providing informed consent to be interviewed between March 2016 and January 2017. Of the seven, three worked in Central Australia and four in the Top End; six were women; six had worked in their current role for more than 1 year, while one had started the role 8 months prior to the interview but had previously worked in a specialist sexual health clinic for more than 1 year. Coordinators worked with between 4 and 11 services, and one also had a region-wide function. None were Aboriginal.

\section{Data collection}

A semi-structured qualitative interview guide was developed to explore participants' understandings of CQI for sexual health; the post-STRIVE trial transition; factors influencing the uptake and sustained application of CQI approaches; and perceptions of the impact of CQI on sexual health service provision. Interviews lasted between 25 and 80 minutes, and were conducted by three interviewers $(J B, P G, S B)$ in person $(n=2)$ or by telephone $(\mathrm{n}=5)$.

\section{Patient and public involvement}

Patients and the public were not involved in this study.

\section{Research team and reflexivity}

Interviews were conducted by $\mathrm{PG}, \mathrm{JB}$ and $\mathrm{SB}$, who had no prior relationship with interviewees in this study. With extensive qualitative research experience, these researchers were able to build rapport and conduct semi-structured interviews with health professionals to elicit deep insight from research participants. The make-up of the research and authorship team also enhanced the credibility of the findings. For instance, all interviewers $(\mathrm{PG}, \mathrm{JB}, \mathrm{SB})$ conducted rigorous qualitative analysis to ensure that agreement was sought on the main findings from the analysis. All other authors have career experience in research and clinical practice focussing on sexual health CQI in diverse Australians settings, which ensured interpretation of data informed by current and historical sexual health CQI practice and policy in the NT. 


\section{Data analysis}

Interviews were audio-recorded and transcribed verbatim before uploading into QRS NVivo V.11 for analysis (QRS International, Melbourne, Australia). A thematic analysis approach ${ }^{20}$ was used by two team members (PG and JB). First, we familiarised ourselves with the data through reading and re-reading of transcripts, noting down initial thematic ideas and 'theoretical memos' ${ }^{20}$ as analytical reminders for making links between different findings. Second, a codebook consisting of parent and child nodes was developed using inductive (codes from initial reads of the interview data) and deductive (codes from existing literature and the discussion guides) data categorisation techniques. Third, all transcripts were coded systematically, while reviewing and editing the codebook according to the data.

\section{RESULTS}

\section{Understanding of sexual health CQI}

Participants consistently described CQI for improved sexual health service delivery as an iterative, ongoing process used to assess current performance of a service, identify strengths and weaknesses, design strategies to improve performance and review data to evaluate the impact of those strategies.

You make a plan, you do the intervention, then you review what you've done, and then you act and improve the deficiencies, or you go with the strengths and try and address the weaknesses. (SH3, Central Australia)

All participants felt that CQI activities could lead to a higher quality of sexual health care by helping staff to understand who is at risk and to identify gaps in service delivery. They also felt that the data collected for sexual health CQI could drive improvements by allowing services to see how they compare and to gauge their performance independent of targets.

The clinics don't know the other clinics on [the list]. They just see where they rank and it makes it pretty evident of how well they're doing... and just focusing the discussion on missed opportunities. (SH2, Central Australia)

\section{Current sexual health CQI practice}

Sexual health coordinators reported varying levels of sexual health CQI engagement and capability in the services they worked with. At one end of the spectrum were services which regularly reviewed data and deployed strategies to address gaps and improve key indicators, such as STI testing. For these services, capability in sexual health CQI appeared to be part of a broader capability and interest in improving performance across different aspects of care:

[Certain services are] always working to their performance indicators... they're always checking on the traffic light reports to see whether they've got their adult health checks done... we just recently realised there was a gap between the 15 to 24 year olds so we've gone through the communities and done some targeted screening. (SHC7, Top End)

In contrast, other services were perceived as undertaking less sexual health CQI activity, or as requiring support from a sexual health coordinator to do so.

They need constant prompts, because what you find is if you go out there, their rates of testing have picked up. That's what happens, when you go out and give them some education, their testing will pick up. You can't take your eye off the ball. (SHC5, Top End)

Higher levels of sexual health CQI capability were attributed to greater managerial support, and CQI data and activity being used to identify and act on areas for improvement.

The two [clinics] that I'm thinking of that do well is because their clinic manager is really on to it and made it a priority. They've been around for a while, those clinic managers. (SH2, Central Australia)

\section{CQI activities, tools and related actions for STI control}

Incorporation of the STRIVE CQI approach into the sexual health coordinators' roles included the use of specific tools-described in detail elsewhere ${ }^{1416}$ - particularly in relation to increasing testing. In summary, these tools included audits, systems assessments, data review and action plans to identify specific activities to improve gaps in service delivery. In addition, 'traffic light reports' is a management tool used by the NT government which draws automated extraction of data from the Primary Care Information System (PCIS) used by government services to report quarterly on selected clinical performance indicators. One participant noted that visual data reports were particularly appreciated by clinic staff.

I've done the stats, my system assessments tools ... that's a very powerful tool because the clinic will sit back and go, 'oh, wow'... They'll look at the spider graphs and they really get into that visual stuff to find out their strengths and weaknesses and how they can improve. (SH5, Top End)

Participants felt the CQI process should identify simple but reproducible actions to improve care. To increase testing and re-testing, some participants cited specific actions including integration of sexual health into routine adult health checks, the use of recalls and reminder cards and prompts embedded in the electronic health information system.

Now that sexual health is integrated into the adult health check, through the men's and women's checks, that has been the single most [important change]. Putting in recalls for sexual health in PCIS has actually improved it. Making sure that I'm auditing so 
that we know if somebody's not being followed up. Then we ask the hard questions why and follow that through. (SH7, Top End)

There's things like putting recalls on for test for re-infection, using the STI template which is embedded in PCIS and things like that as a prompt to help better sexual health delivery. (SH4, Top End)

One participant reported that services engaged in activities such as regular monitoring of individual migration within and between communities, in order to be more responsive to their clients' needs for sexual health care. While this element of patient-centred care does not fit the definition of CQI, the co-ordinator considered this to be closely related to a CQI activity that helps to identify gaps in service delivery.

[Service staff] know to keep an eye out for those people that have been out in the long grass [away from community for a period of time] and coming back to community. That's that undercurrent of stuff that goes on that we talk about and make sure you're checking, that sort of non-documented CQI that you do without doing CQI. (SH7, Top End)

Some participants reported that tools such as the STRIVE systems assessment required adaptation for ongoing use. One co-ordinator noted the increased demand for this type of assessment across different aspects of care, and reluctance among clinic managers to allow staff to participate in what, during the STRIVE trial, were perceived to be lengthy assessment meetings.

Now every program in the NT has a systems assessment attached to it. When we went to do it [systems assessment] last year my boss said to me 'No, we're not', they kept cancelling it. And I said 'Look, why don't we do it, why don't you allow us to have half an hour?' And they said 'Yeah, alright, we can let you have half an hour'. And in that time we did the system assessment. It was quick and really targeted. (SH7, Top End)

\section{Current challenges to implementation of CQI for sexual health Prioritisation of sexual health CQI}

The significant workload associated with acute and chronic conditions in the patient population ${ }^{52122}$ meant primary care staff were not able to prioritise sexual health. This impacted both on sexual health activities within daily clinic practice, and on the time available for CQI during sexual health coordinator visits.

Clinics are bombarded by everything. ...STIs, I mean it's mostly asymptomatic, probably not going to cause too much trouble... it's not a huge priority in engagement in Indigenous people. (SH2, Central Australia)

When I'm out in the community I'll give a talk, depending on how much time I have. Sometimes I'm really pressured for time, they'll only allocate me so much time depending what's going on in the community or in the clinic. (SH5, Top End)

Some participants reported the substantial work in surveillance and response as a result of an outbreak of infectious syphilis, ongoing since 2013 in remote communities in the NT. ${ }^{23}$ However, one participant felt the outbreak increased the perceived importance of sexual health and the use of CQI to drive STI testing and follow-up.

We have ... reports where we can pull out who in the community hasn't had an STI check in the last 12 months, and who hasn't had a syphilis check. Those have been extremely valuable... It gives [clinic managers] a focus, like it gives them people that they can actually go and [test], rather than saying, 'Look, you've only tested $40 \%$ of your people' (SH2, Central Australia)

\section{Lack of staff and staff turnover}

Staff shortages and high staff turnover were mentioned by most participants as presenting a significant challenge to ongoing quality sexual health CQI.

I will go [to a clinic] and do the action plan, and then I'll go back the next time and there'll be new staff, and they won't know what you're talking about. (SH5, Top End)

One participant reported that the lack of Aboriginal health staff increasingly affects the ability to provide high-quality, culturally appropriate health services. It was perceived as important to recruit, train and support younger Aboriginal community members to work in health services, particularly as community members who have been performing these functions retire.

You're finding less and less Indigenous staff in the health centres. Less [Aboriginal] health practitioners. New [non-Aboriginal] staff, even if they're good and conscientious and want to do everything right, still have difficulty with communication, going to different areas. They don't know the people. Some of them aren't there long enough to build up that rapport with people and get their trust. (SH6 Top End)

\section{Future considerations in sexual health CQI Local ownership of CQI processes}

Some participants believed that, while coordinators facilitated the process, sexual health CQI processes should ultimately be owned by staff and managers at primary care services; and could be used to advocate for additional resources to improve sexual health service delivery.

[Our role is] a facilitation role. You're allowing that information and asking the right questions to draw out more answers, being a bit directive but allowing the clinicians to come up with it themselves so they have ownership of it. (SH4, Top End) 
If sexual health isn't a priority because they've only had one staff and they're doing emergencies all day, they can turn around to management and say, 'Yeah, sure, give me more bloody staff', and it's a tool for them too. (SH3, Central Australia)

The level of facilitation required from sexual health coordinators to sustain sexual health CQI was perceived by some participants as dependent on the clinic and clinic manager.

For some communities [clinics] I don't think they need it [facilitation and support to conduct CQI] as much as others, and again, I think that's really dependent on the actual community [clinic] and on the clinic manager. (SH2, Central Australia)

\section{Competing CQI demands}

Participants discussed the tension between maintaining sexual health-specific CQI efforts or integrating them into broader CQI practices. ${ }^{13} 24$

Everyone works and audits and does their quality improvement in such silos that there really seems to be no integration of services talking to each other. (SH2, Central Australia)

Some participants reported that separating sexual health CQI from broader CQI efforts creates confusion and increases the burden on services involved in multiple systems and processes. However, most participants also felt that sexual health-specific CQI, integrated within existing primary care systems and processes, was critical to ensure sexual health is addressed. Without specific processes, sexual health could easily be overlooked due to other service priorities, and because of patient and health worker discomfort.

I do think having a dedicated sexual health team looking at CQI does bring it into focus and I think certainly does engage practitioners to reflect on, you know, how well they do incorporate sexual health practices into their everyday practice. (SH1, Central Australia)

...it's like a big shame job for everyone. Like clinicians are uncomfortable to do it, patients are uncomfortable, so I think it needs to be highlighted. (SH4, Top End)

\section{Information systems and CQI}

Understanding and communicating service activity data was perceived by participants as central to motivating action to improve service delivery. The current patient information management system used by government health services in the NT was perceived to have improved, particularly with respect to automated reporting. However, some participants suggested that the system could be simpler and more responsive to changes in clinical guidelines.
I mean health nowadays is so changeable, you know we're coming up with different testing abilities, different... I think you have to have an information system that can keep up, be flexible and that can change. PCIS was a bit difficult at times to navigate around and get things changed. (SH1, Central Australia)

One participant stressed that information management systems should not just produce aggregated reports but also have capacity to be used to support clinicians in individual patient management, by allowing clinic staff to identify which patients were not being followed up for treatment or recalled for re-testing, for example.

You can turn around and you can say, 'Right, well this is the reason that we're not performing because half the people aren't actually living in the community'... we should be able to get a list of the patients easily and be able to go out to the clinics and give them information, and if need be, do the targeted quality audits that can actually drill in and make clear changes. (SH3, Central Australia)

\section{DISCUSSION}

Our study contributes to existing research on sexual health and CQI in remote Australian settings ${ }^{11} 16212526$ by identifying factors at clinic and Territory levels that both improve and impede the uptake and sustainability of sexual health CQI practices.

At the clinic level, an important factor perceived as enhancing the uptake and sustainability of sexual health CQI was the adaptation of STRIVE CQI tools and reports for use in specific clinic contexts, and for responding to a risk environment such as the syphilis outbreak. The need for adaptation of CQI activities to ensure they are continually fit for purpose has been documented elsewhere. ${ }^{727}$ This study also identified activities that are not traditionally defined as clinical CQI, such as monitoring movement between and within communities, but which assisted service practitioners with monitoring progress and implementing new strategies to improve sexual health outcomes.

Two other clinic-level factors that support the implementation and sustainability of sexual health CQI included local ownership of, and management support for, these processes. However, these were enabled by two regional level factors. The first was regional facilitation of ongoing clinic-level sexual health CQI by sexual health coordinators which helped to raise the profile of sexual health within services, including with managers. Previous research has illustrated the pivotal role of a regional sexual health coordinator function in effective STI interventions in remote Aboriginal communities. ${ }^{16} 28$ The second was the positive framing of CQI as a tool to identify and act on areas for improvement. At the Territory level, ongoing general CQI implementation in the NT is 
attributed to high levels of policy support compared with other states in Australia. ${ }^{27}$

Our study also identified factors that impede the implementation and sustainability of sexual health CQI. As identified in other Australian studies, ${ }^{5} 162122$ participants described how the significant demands of acute and chronic care on Aboriginal primary care services render sexual health CQI a relatively low priority. As has occurred with chronic disease ${ }^{29}$ targets could be used to encourage a greater focus on sexual health. Exploring team structures and work-flow strategies which allow sufficient focus on preventive care ${ }^{30}$ to reduce the likelihood of sexual health being continually overshadowed by more immediate priorities has also been suggested as a strategy to deal with this challenge. ${ }^{31}$

Workforce shortages and high staff turnover were also described as limiting ongoing staff participation in sexual health CQI activities, which in turn inhibits the sustainability of sexual health CQI at a regional level. The lack of experienced Aboriginal health staff was highlighted as particularly problematic, due to the strength of community relationships and trust that Aboriginal health workers and practitioners can build. ${ }^{22}{ }^{31}$ These issues have been documented elsewhere as barriers to the provision of quality care in services that provide health care to Aboriginal populations, ${ }^{16212531}$ and may be particularly important in delivery of quality sexual health care. Strategies to address barriers to Aboriginal workforce participation have the potential to have positive effects on sexual health CQI in these communities and should be supported.

Finally, our findings point to three considerations affecting the future sustainability of sexual health CQI. First, while coordinators explained that sexual health-specific CQI ensured a focus on this difficult health area, ${ }^{521} 22$ they acknowledged the need to reduce the burden on clinics from multiple CQI programmes. Ongoing monitoring of recently available Territory-wide sexual health indicators will help understand if this type of measure is sufficient to increase the perceived importance of sexual health and STI control within primary health care clinics. However, there is also a need to identify which specific CQI tools and activities could be incorporated into generic CQI processes to ensure that sexual health is not overlooked.

Second, findings stress the important contribution of regional sexual health coordinators and support structures to ongoing service improvements and CQI participation in the NT, particularly in the context of high staff turnover. Re-assessment of how support for sexual health CQI is funded and managed may be indicated, and whether this is incorporated within infectious disease surveillance and response, as is largely the case now, or more broadly as part of primary care service development.

Third, access to and use of high-quality information systems and data was seen as a major strength of sexual health CQI, which in turn increases the sustained use of sexual health CQI into the future. The use of benchmarking to motivate action and improvement by allowing services to compare themselves to others is similar to findings from other research. ${ }^{6} 11163132$ The current information system used by government clinics was perceived to be largely supportive of CQI efforts, including the regular review of data, though simplification and ongoing flexibility were recommended. This points to the need for an evolving and responsive health information system.

\section{Study limitations}

There are several limitations to this study. First, this is a qualitative study with a small, non-random sample size, and as such findings should not be viewed as generalisable to all remote settings. Despite the small sample size, 'external validity ${ }^{33}$ - the degree to which findings can be generalised across diverse settings within the NTwas enhanced by interviewing the majority ( 7 of 11) of employees responsible for supporting sexual health CQI within government-led primary health care services in this region. It was also enhanced through data interpretation processes that involved researchers and clinicians with ongoing involvement and interest in CQI, who were able to reflect critically on the findings in the context of current and historical sexual health CQI practice and policy in the NT and other settings in Australia.

Second, the data collection strategies-involving three interviewers, and conducting interviews both in person and by telephone-may have increased the variation among individual responses. However, internal reliability' ${ }^{33}$ was enhanced by interviewers working together to ensure rigour and consistency in data analysis and agreement about the presentation of research findings. Third, given the focus on government-run services, findings may not be generalisable to ACCHS. As part of STRIVEplus, further qualitative research focused on Territory-level key informants and health service staff, across both the government and the community-controlled sectors, will address these gaps.

\section{CONCLUSION}

$\mathrm{CQI}$ is only one of several strategies needed to reduce high STI incidence and prevalence among Aboriginal Australians in remote communities. To ensure sustainability at the service and state level, sexual health CQI activities require responsive and efficient information systems, and, in the context of high staff turnover, a regional coordination function. Strategies to address barriers to the uptake and sustainability of sexual health CQI must be developed and evaluated to reduce the high burden of disease associated with STIs in this population.

\section{Author affiliations}

${ }^{1}$ Kirby Institute, UNSW Sydney, Sydney, New South Wales, Australia

${ }^{2}$ The George Institute for Global Health, UNSW Sydney, Sydney, New South Wales, Australia

${ }^{3}$ Menzies School of Health Research, Brisbane, Queensland, Australia

${ }^{4}$ Burnet Institute, Melbourne, Victoria, Australia 
${ }^{5}$ University Centre for Rural Health, The University of Sydney, Lismore, New South Wales, Australia

${ }^{6}$ South Australian Health and Medical Research Centre, Adelaide, South Australia, Australia

${ }^{7}$ Robinson Research Institute, University of Adelaide, Adelaide, South Australia, Australia

${ }^{8}$ School of Medicine and Public Health, University of Newcastle, Newcastle, New South Wales, Australia

${ }^{9}$ Pacific Clinic Newcastle, HNE Sexual Health, Newcastle, New South Wales,

Australia

${ }^{10}$ Melbourne Sexual Health Centre, Melbourne, Victoria, Australia

${ }^{11}$ Central Clinical School, Monash University, Melbourne, Victoria, Australia

${ }^{12}$ Sydney Sexual Health Centre, Sydney, New South Wales, Australia

${ }^{13}$ Aboriginal Medical Services Alliance Northern Territory, Darwin, Northern Territory, Australia

${ }^{14}$ Centre for Social Research in Health, UNSW Sydney, Sydney, New South Wales, Australia

Contributors PG, GS, JB and SB designed the study. PG, JB and SB conducted data collection. PG, JB and $S B$ undertook analysis of the data. PG, GS and $S B$ led on drafting of the manuscript. All other authors (LM, RB, JW, RG, AR, NR, CKF, BD, $\mathrm{LM}, \mathrm{JK}$ ) assisted with interpretation of data and review of successive drafts of the manuscript. All authors read and approved the final manuscript.

Funding The STRIVEplus study is funded through a National Health and Medical Research Council Partnership Grant (APP1060471).

Disclaimer The funder played no role in design or implementation of this study.

Competing interests The authors have ongoing involvement in Aboriginal health, sexual health and CQI in Australia, as researchers, clinicians and policymakers.

Patient consent for publication Not required.

Ethics approval Approval was obtained from Central Australian Human Research Ethics Committee (HREC 15-298) and the Human Research Ethics Committee of the NT Department of Health and the Menzies School of Health Research (HREC 2015-2374).

Provenance and peer review Not commissioned; externally peer reviewed. Data sharing statement Not applicable as this is a qualitative research.

Open access This is an open access article distributed in accordance with the Creative Commons Attribution Non Commercial (CC BY-NC 4.0) license, which permits others to distribute, remix, adapt, build upon this work non-commercially, and license their derivative works on different terms, provided the original work is properly cited, appropriate credit is given, any changes made indicated, and the use is non-commercial. See: http://creativecommons.org/licenses/by-nc/4.0/.

\section{REFERENCES}

1. Silver BJ, Guy RJ, Wand H, et al. Incidence of curable sexually transmissible infections among adolescents and young adults in remote Australian Aboriginal communities: analysis of longitudinal clinical service data. Sex Transm Infect 2015;91:135-41.

2. Central Australian Rural Practitioners Association. CARPA Standard Treatment ManualAlice Springs: Central Australian Rural Practitioners Association Inc. 2017. 7th edn. validated 29 January 2018. https:// www.remotephcmanuals.com.au/home.html

3. Centre for Disease Control DoH, Northern Territory. NT Guidelines for the Management of Sexually Transmitted Infections in the Primary Health Care Setting. 4th edn: Department of Health, 2016. validated 29 Jan 2018. http://digitallibrary.health.nt.gov.au/prodjspui/handle/ $10137 / 699$

4. Hengel $\mathrm{B}$, Wand $\mathrm{H}$, Ward J, et al. Patient, staffing and health centre factors associated with annual testing for sexually transmissible infections in remote primary health centres. Sex Health 2017:14:274-81.

5. Bailie RS, Si D, O'Donoghue L, et al. Indigenous health: effective and sustainable health services through continuous quality improvement. Med J Aust 2007:186:525-7.

6. Ferlie EB, Shortell SM. Improving the quality of health care in the United Kingdom and the United States: a framework for change. Milbank Q 2001;79:281-315.

7. Lau R, Stevenson F, Ong BN, et al. Achieving change in primary care--causes of the evidence to practice gap: systematic reviews of reviews. Implement Sci 2016;11:40.
8. Grol R, Grimshaw J. From best evidence to best practice: effective implementation of change in patients' care. Lancet 2003;362:1225-30.

9. McElwaine KM, Freund M, Campbell EM, et al. Systematic review of interventions to increase the delivery of preventive care by primary care nurses and allied health clinicians. Implement Sci 2016;11:50.

10. Tricco AC, Ivers NM, Grimshaw JM, et al. Effectiveness of quality improvement strategies on the management of diabetes: a systematic review and meta-analysis. Lancet 2012;379:2252-61.

11. Gardner KL, Dowden M, Togni S, et al. Understanding uptake of continuous quality improvement in Indigenous primary health care: lessons from a multi-site case study of the Audit and Best Practice for Chronic Disease project. Implement Sci 2010;5:21.

12. Gibson-Helm ME, Teede HJ, Rumbold AR, et al. Continuous quality improvement and metabolic screening during pregnancy at primary health centres attended by Aboriginal and Torres Strait Islander women. Med J Aust 2015;203:369-70.

13. Allen and Clarke. Evaluation of the Northern Territory Continuous Quality Improvement (CQI) Investment Strategy: Summary report. Department of Health, Canberra 2016.

14. Ward J, McGregor S, Guy RJ, et al. STI in remote communities: improved and enhanced primary health care (STRIVE) study protocol: a cluster randomised controlled trial comparing 'usual practice' STI care to enhanced care in remote primary health care services in Australia. BMC Infect Dis 2013;13:425.

15. Ward J, Guy RJ, Rumbold AR, et al. A pragmatic stepped wedge cluster randomised trial of continuous quality improvement strategies to improve the control of sexually transmissible infections in remote Australian Aboriginal communities. forthcoming.

16. Hengel B, Bell S, Garton L, et al. Perspectives of primary health care staff on the implementation of a sexual health quality improvement program: a qualitative study in remote aboriginal communities in Australia. BMC Health Serv Res 2018;18:230.

17. O'Brien BC, Harris IB, Beckman TJ, et al. Standards for reporting qualitative research: a synthesis of recommendations. Acad Med 2014;89:1245-51.

18. Australian Bureau of Statistics. 2016 Census QuickStats - Northern Territory, 2016. (validated 9 August 2018). http://quickstats. censusdata.abs.gov.au/census_services/getproduct/census/2016/ quickstat/7?opendocument.

19. Northern Territory Department of Health. 2016-17 Annual Report: NT Health, 2017. (verified 29 January 2018). http://digitallibrary.health.nt. gov.au/prodjspui/handle/10137/1417

20. Strauss A, Corbin J. Basics of qualitative research. Thousand Oaks: Sage, 1990

21. Hengel B, Maher L, Garton L, et al. Reasons for delays in treatment of bacterial sexually transmissible infections in remote Aboriginal communities in Australia: a qualitative study of healthcentre staff. Sex Health 2015;12:341-7.

22. Newham J, Schierhout G, Bailie R, et al. 'There's only one enabler; come up, help us': staff perspectives of barriers and enablers to continuous quality improvement in Aboriginal primary health-care settings in South Australia. Aust J Prim Health 2016;22:244-54.

23. Bright A, Dups J. Infectious and congenital syphilis notifications associated with an ongoing outbreak in northern Australia. Commun Dis Intell Q Rep 2016;40:E7-10.

24. The Lowitja Institute. Recommendations for a National CQ Framework for Aboriginal and Torres Strait Islander Health, 2014. (verified 29 January 2018). http://www.health.gov.au/internet/main/ publishing.nsf/content/cqi-framework-atsih.

25. Schierhout G, Hains J, Si D, et al. Evaluating the effectiveness of a multifaceted, multilevel continuous quality improvement program in primary health care: developing a realist theory of change. Implement Sci 2013;8:119.

26. Gardner K, Bailie R, Si D, et al. Reorienting primary health care for addressing chronic conditions in remote Australia and the South Pacific: review of evidence and lessons from an innovative quality improvement process. Aust J Rural Health 2011;19:111-7.

27. Woods C, Carlisle K, Larkins S, et al. Exploring Systems That Support Good Clinical Care in Indigenous Primary Health-care Services: A Retrospective Analysis of Longitudinal Systems Assessment Tool Data from High-Improving Services. Front Public Health 2017:5

28. Guy R, Ward JS, Smith KS, et al. The impact of sexually transmissible infection programs in remote Aboriginal communities in Australia: a systematic review. Sex Health 2012;9:205-12.

29. Bailie J, Schierhout GH, Kelaher MA, et al. Follow-up of Indigenousspecific health assessments - a socioecological analysis. Med J Aust 2014;200:653-7.

30. Nattabi B, Girgis S, Matthews V, et al. Clinic predictors of better syphilis testing in Aboriginal primary health care: a promising 
opportunity for primary health care service managers. Aust $\mathrm{J}$ Prim Health 2018:350.

31. Bailie J, Matthews V, Laycock A, et al. Improving preventive health care in Aboriginal and Torres Strait Islander primary care settings. Global Health 2017;13:48.
32. Larkins S, Woods CE, Matthews V, et al. Responses of Aboriginal and Torres Strait Islander Primary Health-Care Services to Continuous Quality Improvement Initiatives. Front Public Health 2015;3:288.

33. Bryman A. Social Research Methods. 5th edn. Oxford University Press: Oxford, 2016. 